\title{
Eighteen year weight trajectories and metabolic markers of diabetes in modernising China: which timescale is most relevant? Reply to Vistisen D and Færch K [letter]
}

\author{
Annie Green Howard • Penny Gordon-Larsen • Elizabeth Koehler • \\ Lauren Paynter • Amanda L. Thompson • Linda S. Adair • \\ Bing Zhang • Amy H. Herring
}

Received: 27 August 2014 / Accepted: 27 August 2014 / Published online: 30 September 2014

(C) Springer-Verlag Berlin Heidelberg 2014

Keywords Fasting glucose $\cdot$ Insulin resistance $\cdot$ Latent class trajectory analysis $\cdot$ Weight trajectories

\section{Abbreviation \\ CHNS China Health and Nutrition Survey}

To the Editor: Vistisen and Færch [1] make an interesting point regarding the choice of timescale used to fit trajectory models. The authors assert that time since diagnosis would be the appropriate timescale when studying the aetiology of diabetes development or prognosis of diabetes, and we agree that this would be preferred. However, there are some practical issues for defining the appropriate time point of reference relative to diagnosis of disease in a population-based study,

A. G. Howard · E. Koehler · A. H. Herring

Department of Biostatistics, Gillings School of Global Public Health, University of North Carolina, Chapel Hill, NC, USA

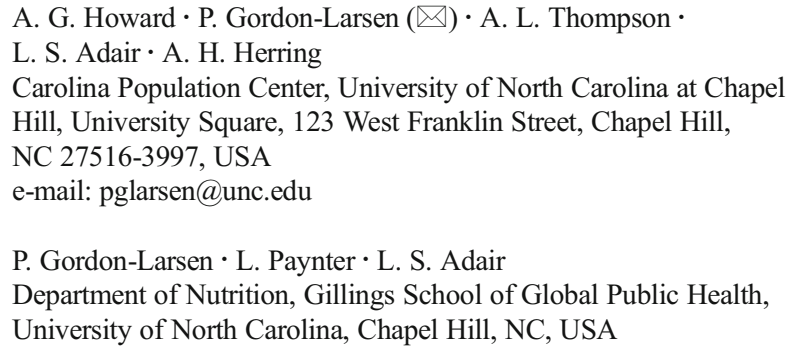

such as ours [2]. Examining trajectories up to the time of diagnosis would be ideal. However, it is hard to know the exact timing of diagnosis, since the collection of fasting blood and assays for cardiometabolic biomarkers were completed only in 2009 and therefore we have no data on comparable measures prior to 2009, which would be necessary to establish time of diagnosis. Data from the China Health and Nutrition Survey (CHNS) suggest that in this population the majority of diabetes is undiagnosed (over 50\%) [3], and therefore it will be less likely to influence weight trajectories. All that being said, we acknowledge that our method, using time since enrolment could, as the authors suggest, introduce errors that may bias results. This is a testable hypothesis that could be addressed through a simulation study to see what types of inferences one could make using a variety of different approaches to handle time. Indeed, we would welcome the idea of such a future study and are currently investigating related issues using simulation studies. Additionally, future studies involving CHNS data - at which point we will have multiple measurements of fasting blood and assays for cardiometabolic biomarkers - will allow us to additionally investigate this question further.

Duality of interest The authors declare that there is no duality of interest associated with this manuscript.

Contribution statement AGH, AHH, LSA and PGL drafted the letter and all authors reviewed and approved the final version.

\section{References}

1. Vistisen D, Færch K (2014) Eighteen year weight trajectories and metabolic markers of diabetes in modernising China: which time scale is most relevant? Diabetologia. doi:10.1007/s00125-014-3391-9 
2. Gordon-Larsen P, Koehler E, Howard AG et al (2014) Eighteen year weight trajectories and metabolic markers of diabetes in modernising China. Diabetologia 57:1820-1829, erratum in $57: 2005$
3. Attard SM, Herring AH, Mayer-Davis EJ, Popkin BM, Meigs JB, Gordon-Larsen P (2012) Multilevel examination of diabetes in modernising China: what elements of urbanisation are most associated with diabetes? Diabetologia 55:3182-3192 